\title{
ORIGINAL
}

\section{Survival benefit of extracorporeal membrane oxygenation in severe COVID-19: a multi-centre-matched cohort study}

\author{
Stephen Whebell ${ }^{1,6^{*}}{ }^{1}$, Joe Zhang ${ }^{2,3}$, Rebecca Lewis ${ }^{1}$, Michael Berry ${ }^{2}$, Stephane Ledot ${ }^{4}$, Andrew Retter ${ }^{1}$ \\ and Luigi Camporota ${ }^{1,5}$
}

(c) 2022 Springer-Verlag GmbH Germany, part of Springer Nature

\begin{abstract}
Purpose: Extracorporeal membrane oxygenation (ECMO) has become an established therapy for severe respiratory failure in coronavirus disease 2019 (COVID-19). The added benefit of receiving ECMO in COVID-19 remains uncertain. The aim of this study is to analyse the impact of receiving ECMO at specialist centres on hospital mortality.

Methods: A multi-centre retrospective study was conducted in COVID-19 patients from 111 hospitals, referred to two specialist ECMO centres in the United Kingdom (UK) (March 2020 to February 2021). Detailed covariate data were contemporaneously curated from electronic referral systems. We analysed added benefit of ECMO treatment in specialist centres using propensity score matching techniques.

Results: 1363 patients, 243 receiving ECMO, were analysed. The best matching technique generated 209 matches, with a marginal odds ratio $(\mathrm{OR})$ for mortality of $0.44(95 \% \mathrm{Cl} 0.29-0.68, p<0.001)$ and absolute mortality reduction of $18.2 \%$ (44\% vs $25.8 \%, p<0.001)$ for treatment with $E C M O$ in a specialist centre.

Conclusion: We found ECMO provided at specialist centres conferred significant survival benefit. Where resources and specialism allow, ECMO should be widely offered.
\end{abstract}

Keywords: ECMO, COVID-19, ARDS, Severe respiratory failure

\section{Introduction}

Extracorporeal membrane oxygenation (ECMO) has become a widespread therapy for severe respiratory failure (SRF) in coronavirus disease 2019 (COVID-19). While some reports indicate high mortality amongst COVID-19 patients receiving ECMO [1-4], other studies describe improved outcomes $[5,6]$, with a recent review reporting a $37.1 \%$ pooled in-hospital mortality [7]-comparable to pandemic influenza and non-COVID-19 acute respiratory distress syndromes [8]. Consensus guidelines

\footnotetext{
*Correspondence: swhebell@gmail.com

${ }^{6}$ Department of Adult Critical Care, St Thomas' Hospital, London SE1 7EH, UK

Full author information is available at the end of the article
}

have given cautious recommendation for ECMO in selected COVID-19 patients [9, 10]. Limited evidence from causal analyses in cohorts of COVID-19 ECMO patients $[11,12]$ suggests ECMO confers a survival benefit. However, given varied selection criteria, outcomes, and baseline characteristics [7, 13], the added benefit of ECMO in the COVID-19 SRF population remains uncertain.

A centralised national referral system was established at the start of the pandemic, providing a unified pathway for hospitals in the United Kingdom (UK) to refer patients for consideration of ECMO to specialist centres [14]. The high volume of referrals [15], and the detailed data recorded through the referral system, allow analysis of ECMO as an intervention in a large

\section{勿


and well-characterized cohort of patients with COVID19 SRF. In this study, we obtain a dataset of patients referred from 111 hospitals in the UK to two national ECMO centres, serving 24.5 million people in southern England and Wales. We perform a matching analysis to estimate the added survival benefit of receiving treatment via a specialist ECMO service, when compared to patients who received conventional therapy at their referring centre.

\section{Methods}

\section{Study design and setting}

A multi-centre retrospective cohort study was conducted in patients referred to two national ECMO centres [Guy's and St Thomas' Foundation Trust (GSTFT) and Royal Brompton and Harefield Trust (RBHT)] in the UK [16], between 3 March 2020 and 28 February 2021.

Following National Health Service commissioning in 2011, the UK patients with SRF may be referred to a designated ECMO centre, where specialists perform remote assessment, deliver advice, and consider patients against national eligibility criteria for retrieval on mobile ECMO. Criteria are outlined by NHS England (Supplementary Table A), establishing consistent indications for respiratory ECMO provision [17]. The primary indication is potentially reversible SRF (as indicated by severe hypoxaemia, Murray-score $>3$, or uncompensated hypercapnia, in absence of significant frailty and organ failure severity).

Pathway changes took place at the start of the pandemic. Patient referrals were delivered through an online 'Refer-a-patient' portal (Bloomsbury Health, London, UK). Eligibility criteria were revised on 10 April 2020 to include consideration of respiratory ECMO survival prediction (RESP) score [14], and again in the latter half of the data collection period to include days of non-invasive ventilation (NIV) [14]. The pathway for COVID-19 ECMO provision has been previously described [6].

\section{Patient selection}

All referred patients with COVID-19 were eligible for inclusion. UK practice encourages active referral and discussion [6]. This approach includes patients when referring clinicians considered them to have COVID-19 SRF requiring specialist input. In a subset of patients referred twice (following closure of initial referral), the second referral instance was eligible for inclusion, as only the second could result in ECMO retrieval.

Patients referred with a non-COVID-19 diagnosis, or those referred for non-respiratory ECMO, were excluded. Patients with missing outcome data were excluded.

\section{Take-home message}

Extracorporeal membrane oxygenation (ECMO) has been used widely throughout the coronavirus disease 2019 (COVID-19) pandemic, however, conflicting outcomes have been reported. We found that in a well-matched cohort of ECMO-referred patients, retrieval on mobile ECMO to a specialist center conferred survival benefit.

\section{Study exposure}

In the UK pandemic ECMO provision, referred and accepted patients are retrieved on mobile ECMO to a specialist centre (treatment group). Our exposure is therefore two-pronged, including provision of ECMO, and specialist SRF care in the centre itself. The control group contains patients whose referrals were not accepted, remaining in referring centres receiving conventional care.

This on-going practice of mobile ECMO retrieval has removed separation between transfer to specialist centres, and ECMO initiation, described in Peek et al. [18], where patients were transferred conventionally, with only a subset receiving ECMO.

\section{Patient outcomes}

Our primary outcome measure was in-hospital mortality during the same hospital episode as the referral. We treated inter-hospital transfers or repatriations as continuing the same hospital episode. There were no secondary outcome measures.

\section{Data collection and curation}

Demographic, clinical, and physiological parameters were submitted by the referring team at time of referral via the 'Refer-a-patient' system. Referral data were immediately reviewed by the designated ECMO centre, and following consideration, electronic responses were returned, documenting clinical decision (retrieve, manage at referring hospital, or perceived futility), and ECMO eligibility status by NHS criteria. A decision of 'futility' indicated an illness trajectory that was deemed to have limited reversibility with addition of ECMO. In the absence of validated scoring systems, these decisions were undertaken in discussion with specialists in referring and ECMO teams.

A bespoke data extraction pipeline was created in Python (Python Software Foundation, https://www. python.org/) to curate data into a structured database, ensure consistency and excluding erroneous values. Parallel manual cross-checking was employed to ensure data quality. 
Outcome data were collected manually through direct contact with hospital audit, medical records, and clinical departments. Direct follow-up ensured mortality during the relevant hospital episode was identified.

\section{Missing data handling}

A subset of patient referrals was incomplete. We used random forest imputation [19] to handle missingness, subsequently testing this approach in sensitivity analysis.

\section{Statistical analysis}

We described the cohort across two pandemic waves, and separately examined referrals labelled 'perceived futility'. The pandemic waves were defined as occurring before or after 1 August 2020. Continuous variables are presented with median and interquartile range and categorical with count and percentage. Between-groups comparisons are made using Mann-Whitney $U$ for continuous and Pearson's Chi-squared for categorical variables. A $p$ value of $<0.05$ was considered statistically significant.

\section{Propensity score and matching}

To measure the effect of our exposure on the primary outcome, we adopted propensity score (PS) matching. This enabled consideration of numerous covariates as contributors to confounding relative to number of events, and more granular exploration of matched and unmatched populations. Compared to multivariable analysis, matching accounts for changes in case characteristics and decision-making over time, by identifying comparable samples from observational real-world data [20].

To account for confounding from unmeasured discriminators of 'too severe disease' that would preclude ECMO, we excluded patients with a decision of 'perceived futility' from matching.

We additionally considered handling of patients labelled as 'not meeting NHS criteria'. Changes to criteria occurred over the course of the pandemic (see "Study design"), and application of criteria required use of expert clinical judgement in deciding eligibility. Once patients labelled with 'futility' were excluded from the 'not meeting NHS criteria' group, the remainder contained many marginal cases, and the label was not felt to confer clear properties that might confound matching. We included this group for the main analysis, and tested assumptions in sensitivity analysis.

PS for treatment with ECMO was constructed using multivariable logistic regression. Covariates for PS generation (Supplementary Table F) were chosen based on their consideration in decision-making, and representation of severity.
Patients retrieved on mobile ECMO were matched with referred patients managed conventionally at referring centres. Three different matching techniques were evaluated: nearest neighbour calliper matching, genetic matching (GenMatch) [21], and optimal pair matching. A calliper width of 0.1 standard deviations of the logit of the PS was used for nearest neighbour and GenMatch. All matching was conducted in a 1:1 ratio without replacement. Balance between groups was assessed using standardised mean difference (SMD), with a SMD of $<0.1$ indicating good balance [22].

The marginal odds ratio (OR) for hospital mortality with ECMO in the matched cohorts was estimated using univariable logistic regression. Bias-corrected confidence intervals were calculated using bootstrapping (4999 replications). The $E$ value for each OR estimate of treatment effect was calculated to estimate unmeasured confounding $[23,24]$.

Statistical analysis and matching procedures were performed using R 4.0.3 (R Core Team, 2018) and the Matchit package [25].

\section{Sensitivity analyses}

Sensitivity analysis of PS matching was performed by (1) restricting eligible patients to those documented as 'meeting NHS criteria'; (2) restricting population to patients with $<10 \%$ missing data. The matching procedure which generated the best combination of matches and covariate balance was used.

\section{Ethics and approval}

This study qualified as a service evaluation, as defined by the UK Health Research Authority, with waiver of individual informed consent. Data collection and analysis were carried out under evaluation for national ECMO commissioning.

\section{Results}

\section{Patient characteristics}

During the study period, 1580 patients with COVID-19 SRF were referred from 111 hospitals. Following exclusions, final analysis included 1363 patients, of which 243 were transferred to an SRF centre on mobile ECMO for continued treatment. Figure 1 shows a flow chart of patient inclusion. All patients in the treatment group received veno-venous (vv) ECMO (vv-ECMO). Referrals and outcomes over time are shown in Fig. 2. Prior to imputation, the dataset exhibited $11.1 \%$ missingness (Supplementary Table B, Supplementary Figure A).

Characteristics of the overall population and comparison based on referral decision are presented in Table 1. 430 (31.5\%) patients had 'perceived futility' documented and were excluded from matching analysis. Compared to 


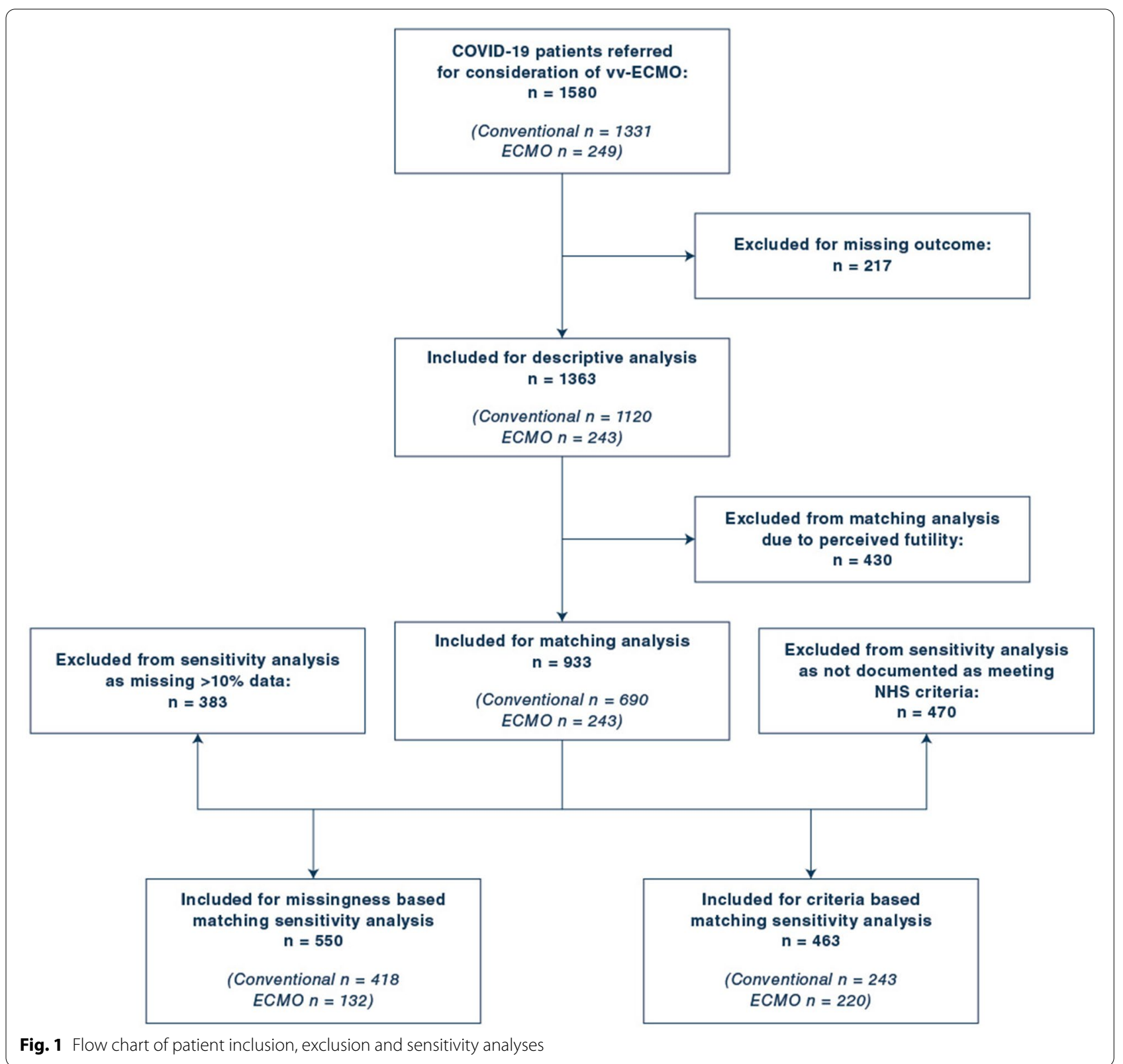

included patients, this group was older, with more severe organ failure, was hospitalised and ventilated for longer, and more likely to die ( $73 \%$ vs $43.2 \%, p<0.001$ ).

Comparisons between two COVID-19 waves are presented in Supplementary Tables C-E. Mortality for conventionally managed referrals increased in wave $2(51.9 \%$ vs $62.4 \%, p=0.001$ ), but remained stable in ECMO-treated patients $(22.9 \%$ vs $26.1 \%, p=0.672)$. Wave 2 patients experienced shorter durations of hospitalisation and IMV prior to referral. They received more immunomodulation, less renal replacement therapy, more NIV, and developed greater barotrauma (with more chest drains). Overall, a greater proportion of patients with documented 'perceived futility' outcomes and a lower proportion of ECMOtreated patients were seen in wave 2 .

\section{Matching and treatment effect of ECMO}

933 patients were available for matching. Nearest neighbour matching generated matches for 208 ECMO-treated patients, with a marginal OR for mortality of 0.47 (95\% CI $0.32-0.73, p<0.001, E$ value $2.28, E$ value lower $C I 1.62)$ and absolute risk reduction (ARR) of $16.3 \%$ (42.3\% vs $26 \%, p=0.001$ ) given ECMO treatment. GenMatch generated matches for 209 ECMO patients, with a marginal 


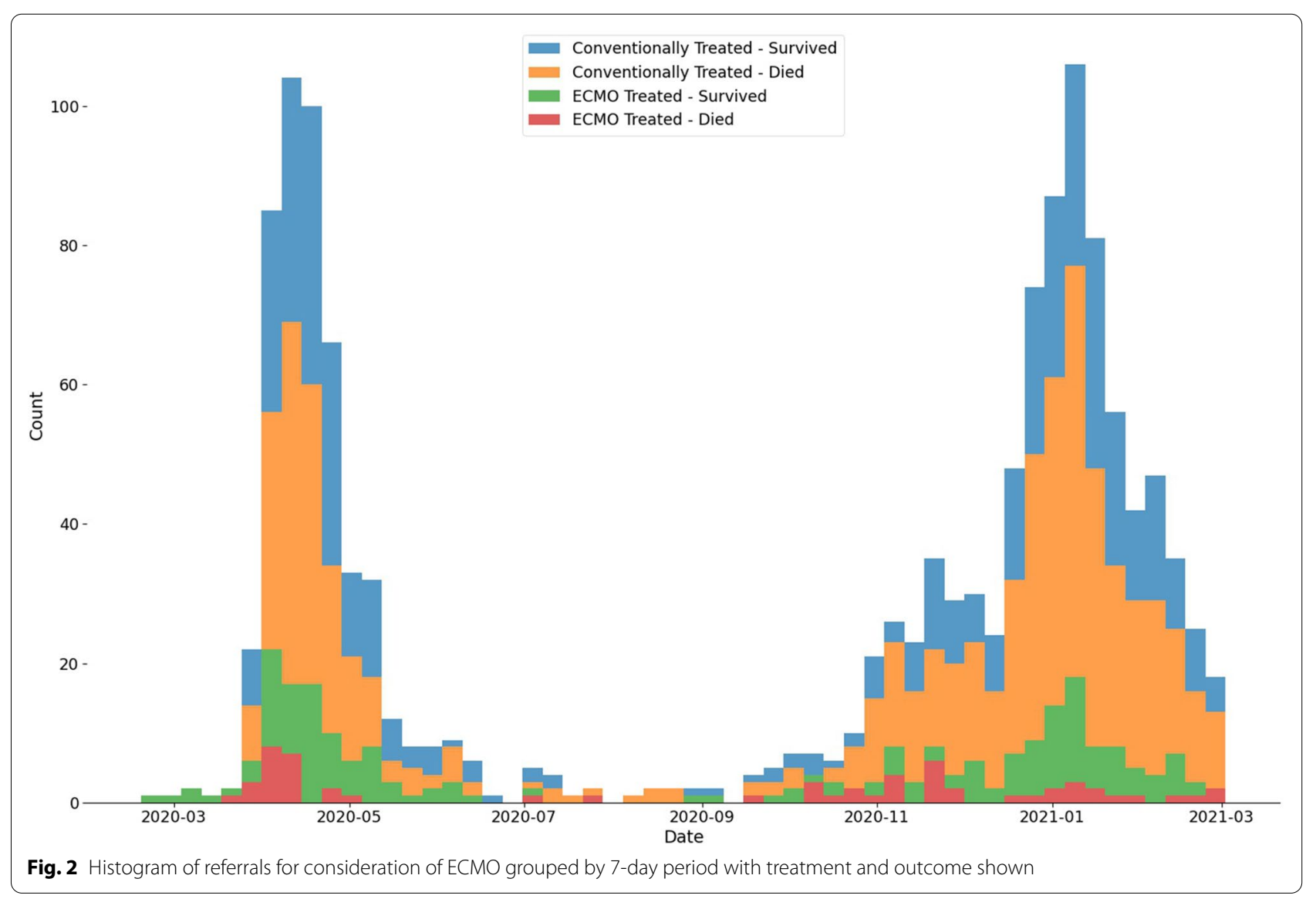

OR for mortality of 0.44 (95\% CI $0.29-0.68, p<0.001, E$ value $2.38, E$ value lower CI 1.72) and ARR of $18.2 \%$ (44\% vs $25.8 \%, p<0.001$ ) given ECMO treatment. Optimal pair matching generated matches for 237 ECMO patients, with a marginal OR for mortality of 0.47 (95\% CI $0.31-$ $0.70, p<0.001, E$ value $2.28, E$ value lower $C I 1.68)$ and ARR of $16.5 \%$ ( $41.4 \%$ vs $24.9 \%, p<0.001)$ given treatment with ECMO.

GenMatch matching resulted in the best combination of matches and covariate balance. Characteristics, outcomes, and standardised mean difference (SMD) of patients matched via this method are presented in Table 2. All covariates had a $\mathrm{SMD} \leq 0.1$ using GenMatch. The 34 ECMO-treated patients who were unable to be matched using this method were younger, had a shorter duration of IMV, were more hypoxic, had higher RESP scores, and received less corticosteroids (Table 3 ).

Characteristics, outcomes, and balance metrics of patients matched via nearest neighbour and optimal pair matching are presented in Supplementary Tables G-I.

\section{Sensitivity analysis}

In sensitivity analysis of selection criteria, a subgroup with 463 patients were documented as 'meeting NHS criteria' for respiratory ECMO (220 conventional, 243 received ECMO). GenMatch generated 138 matches in this restricted cohort. The marginal OR for mortality was 0.52 (95\% CI $0.32-0.89, p=0.012, E$ value $2.12, E$ value lower CI 1.31) with an ARR of $14.5 \%$ (42\% vs $27.5 \%$, $p=0.016)$. Characteristics are shown in Supplementary Table J. Good balance was achieved with an SMD $\leq 0.1$ for all covariates except for $\mathrm{pH}$, which was slightly higher in the ECMO-treated cohort (SMD - 0.11).

In sensitivity analysis for missingness, a subgroup containing 550 patients remained (418 conventional, 132 ECMO) after exclusion for $>10 \%$ of missing data. Matches for 116 patients were generated using GenMatch, with marginal OR for mortality of 0.41 (95\% CI $0.24-0.71, p=0.002, E$ value 2.5 , lower CI 1.66). Good balance was achieved with an SMD of $\leq 0.1$ for all covariates. Characteristics shown in Supplementary Table K.

\section{Discussion}

The use of ECMO for COVID-19 SRF is supported by reports of safety and acceptable outcomes in retrospective cohorts [26], and more limited causal analysis in observational data. Our findings suggest that ECMO 
Table 1 Characteristics, decision and outcomes of COVID-19 patients referred for ECMO

\begin{tabular}{|c|c|c|c|c|}
\hline & $\begin{array}{l}\text { All referrals } \\
(n=1383)\end{array}$ & $\begin{array}{l}\text { Included for matching } \\
(n=933)\end{array}$ & $\begin{array}{l}\text { 'Perceived futility' } \\
(n=430)\end{array}$ & $p$-value \\
\hline \multicolumn{5}{|l|}{ Demographics } \\
\hline Age & $53[45,60]$ & $51[43,58]$ & $58[52,63]$ & $<0.001$ \\
\hline Male sex & 941 (69) & 641 (68.7) & $300(69.8)$ & 0.74 \\
\hline Body mass index $\left(\mathrm{kg} / \mathrm{m}^{2}\right)$ & $31.01[27.02,35.46]$ & $31.35[27.34,35.64]$ & $29.88[26.31,34.64]$ & 0.002 \\
\hline Clinical frailty scale & $2[2,2]$ & $2[2,2]$ & $2[2,2]$ & $<0.001$ \\
\hline Immunocompromised & $82(6)$ & $40(4.3)$ & $42(9.8)$ & $<0.001$ \\
\hline \multicolumn{5}{|l|}{ Pre-referral admission characteristics } \\
\hline Hospital duration prior to IMV (days) & $5[2,8]$ & $4.5[2,7.92]$ & $6.52[3,9.38]$ & $<0.001$ \\
\hline IMV duration ${ }^{a}$ (days) & $3.15[1,6]$ & $3[1,6]$ & $4[2,9]$ & $<0.001$ \\
\hline Cardiac arrest & $43(3.2)$ & $24(2.6)$ & $19(4.4)$ & 0.1 \\
\hline \multicolumn{5}{|l|}{ Therapeutic interventions } \\
\hline Non-invasive ventilation ${ }^{\mathrm{b}}$ prior to IMV & $921(67.6)$ & $612(65.6)$ & 309 (71.9) & 0.025 \\
\hline Corticosteroids & $910(66.8)$ & $606(65)$ & $304(70.7)$ & 0.042 \\
\hline Interleukin-6 inhibitor & $117(8.6)$ & $77(8.3)$ & $40(9.3)$ & 0.59 \\
\hline Neuromuscular blockade & $1178(86.4)$ & $803(86.1)$ & $375(87.2)$ & 0.626 \\
\hline Prone position trial & $1054(77.3)$ & $712(76.3)$ & $342(79.5)$ & 0.211 \\
\hline Inhaled nitric oxide use & $168(12.3)$ & $98(10.5)$ & $70(16.3)$ & 0.003 \\
\hline Chest drain(s) for pneumothorax & $83(6.1)$ & $55(5.9)$ & $28(6.5)$ & 0.749 \\
\hline Renal replacement therapy & $135(9.9)$ & $63(6.8)$ & $72(16.7)$ & $<0.001$ \\
\hline Noradrenaline dose (mcg/kg) & $0.04[0,0.12]$ & $0.03[0,0.1]$ & $0.06[0.01,0.19]$ & $<0.001$ \\
\hline Anticoagulation & & & & $<0.001$ \\
\hline Contraindicated & $11(0.8)$ & $7(0.8)$ & $4(0.9)$ & \\
\hline Prophylactic & $883(64.8)$ & $647(69.3)$ & $236(54.9)$ & \\
\hline Therapeutic & $469(34.4)$ & 279(29.9) & $190(44.2)$ & \\
\hline \multicolumn{5}{|c|}{ Respiratory parameters at time of referral } \\
\hline P/F ratio $(\mathrm{mmHg})$ & $78.33[64.7,97.78]$ & $80[67.28,100.5]$ & $73.93[61.5,91.67]$ & $<0.001$ \\
\hline Static compliance $\left(\mathrm{mL} / \mathrm{cmH}_{2} \mathrm{O}\right)$ & $28.87[20.89,35.71]$ & $30[22,37]$ & $26.4[19.44,33.72]$ & $<0.001$ \\
\hline Vt/PBW (mL/kg) & $6.7[5.98,7.87]$ & $6.74[6.06,7.99]$ & $6.55[5.78,7.61]$ & 0.001 \\
\hline Driving pressure $\left(\mathrm{cmH}_{2} \mathrm{O}\right)$ & $16[13,20]$ & $15.7[13,19]$ & $17[14,22]$ & $<0.001$ \\
\hline Plateau pressure $\left(\mathrm{cmH}_{2} \mathrm{O}\right)$ & $29[25,31]$ & $29[25,31]$ & $29[25,32]$ & 0.065 \\
\hline Positive end expiratory pressure $\left(\mathrm{cmH}_{2} \mathrm{O}\right)$ & $12[10,14]$ & $12[10,14.2]$ & $12[10,14]$ & 0.218 \\
\hline Minute ventilation (L/min) & $9.84[8,11.52]$ & $9.88[8.04,11.5]$ & $9.65[7.92,11.63]$ & 0.715 \\
\hline \multicolumn{5}{|c|}{ Arterial blood gas parameters at time of referral } \\
\hline $\mathrm{pH}$ & $7.31[7.24,7.38]$ & $7.32[7.25,7.39]$ & $7.29[7.2,7.36]$ & $<0.001$ \\
\hline $\mathrm{pCO}_{2}(\mathrm{mmHg})$ & $59.62[50.25,71.03]$ & $58.12[49.2,68.55]$ & $63.9[53.25,77.25]$ & $<0.001$ \\
\hline Bicarbonate (mmol/L) & $27.02[24,31.45]$ & $26.98[24.08,30.75]$ & $27.6[24,32.6]$ & 0.066 \\
\hline Base excess (mmol/L) & $3.73[1.8,7.8]$ & $3.6[1.8,7.4]$ & $4.05[1.8,8.4]$ & 0.219 \\
\hline Lactate $(\mathrm{mmol} / \mathrm{L})$ & $1.3[1,1.8]$ & $1.3[1,1.7]$ & $1.4[1,1.8]$ & 0.111 \\
\hline \multicolumn{5}{|c|}{ Laboratory parameters at time of referral } \\
\hline Haemoglobin $(g / L)$ & $113.55[98,126]$ & $115[101,127]$ & $108.5[92,124]$ & $<0.001$ \\
\hline White cell count (10^9/L) & $12.04[9.31,15.55]$ & $11.86[9.2,15]$ & $12.91[9.8,16.88]$ & 0.001 \\
\hline Platelets $\left(10^{\wedge} 9 / \mathrm{L}\right)$ & $272.1[229,319.91]$ & $278.17[235,325]$ & $263.78[218.14,307.27]$ & $<0.001$ \\
\hline Creatinine $(\mu \mathrm{mol} / \mathrm{L})$ & $73[56,99.63]$ & $71[55,93]$ & $79[59.25,117.5]$ & $<0.001$ \\
\hline C-reactive protein (mg/L) & $165.63[76.5,270.1]$ & $170[80,274]$ & $148.47[68,260]$ & 0.1 \\
\hline \multicolumn{5}{|l|}{ Severity and risk scores } \\
\hline RESP score & $4[2.13,5]$ & $5[3,5]$ & $3[1,4]$ & $<0.001$ \\
\hline SOFA score & $5.90[4,8]$ & $5[4,7]$ & $7[4,8]$ & $<0.001$ \\
\hline \multicolumn{5}{|l|}{ Referral outcomes } \\
\hline Meets NHS criteria & $608(46.8)$ & $463(52.7)$ & $145(34.5)$ & $<0.001$ \\
\hline
\end{tabular}


Table 1 (continued)

\begin{tabular}{|c|c|c|c|c|}
\hline & $\begin{array}{l}\text { All referrals } \\
(n=1383)\end{array}$ & $\begin{array}{l}\text { Included for matching } \\
(n=933)\end{array}$ & $\begin{array}{l}\text { 'Perceived futility' } \\
(n=430)\end{array}$ & $\boldsymbol{p}$-value \\
\hline Perceived futility & $430(31.5)$ & $243(26)$ & $0(0)$ & $<0.001$ \\
\hline Treated with ECMO & $243(17.8)$ & $403(43.2)$ & $314(73)$ & $<0.001$ \\
\hline Died & $717(52.6)$ & $463(52.7)$ & $145(34.5)$ & $<0.001$ \\
\hline
\end{tabular}

Values are expressed as median value $[95 \% \mathrm{Cl}]$ or as $\mathrm{n}(\%) ; p$ values for comparison statistics computed for 'perceived futility' vs other referral decision only

$I M V$ invasive mechanical ventilation, $P / F$ ratio partial pressure of arterial oxygen to fraction of inspired oxygen ratio, $V t$ tidal volume, $P B W$ predicted body weight, $R E S P$ respiratory ECMO survival prediction, SOFA sequential organ failure assessment, NHS national health service

a IMV duration includes all days of IMV, regardless of intensity

b NIV use does not include high flow oxygen therapy

delivery at specialist SRF centres confers significant survival benefit, compared to matched patients who received conventional therapy in referring centres.

The considerable treatment effect (ARR 18.2\%) produced in our study warrants closer examination. This effect was robust through different matching techniques and sensitivity analyses. For comparison, EOLIA [27] found an ARR of $11 \%$ between two arms, but was underpowered with substantial, late cross-over in the control group. Previous commentaries have noted that ARR would be greater if survival of cross-over patients was considered $[28,29]$. The most comparable study from COVID-19 ECMO is Shaefi et al. [12], who adopted a multivariable adjustment approach, finding adjusted hazard ratio of 0.55 for death given ECMO (ARR 12.8\%). In contrast, our results may represent added effect of treatment in a specialist SRF centre. Peek et al. [18] previously demonstrated ARR of $16 \%$ following transfer to a UK SRF centre, but with ECMO provided to only $76 \%$ of patients in the treatment group (as discussed, this separation is not present in standard UK ECMO pandemic pathways, where retrieval on mobile ECMO is usual practice).

Reported outcomes for COVID-19 ECMO remain varied. Recent examination of a large COVID-19 ECMO cohort [4] reported in-hospital mortality of $68 \%$. Mortality in our study is considerably lower than Karagiannidis et al., and is also lower than that reported via international registries $[26,30]$ and previous non-COVID-19 trials [27]. The increase in ECMO mortality over time observed in other studies $[30,31]$ was also not seen in our cohort. Our population differs significantly from other published cohorts. First, while severity of respiratory failure was similar, it is likely that strict criteria enacted during the pandemic resulted in a more "well" population. Compared to published data, this is evidenced by younger age, shorter pre-ECMO ventilation, less renal support, lower SOFA, and higher RESP scores. Application of these criteria appeared consistent over time in our study, with clinical characteristics of ECMO patients remaining similar across waves (barring preECMO therapeutic interventions). Second, all patients were retrieved on ECMO and treated in high-volume ECMO centres, which may associate with improved survival $[26,32]$. These differences may highlight the impact of selective inclusion criteria, and centralised ECMO provisioning structures.

Neither our nor Shaefi et al's approach can negate effects of residual confounding. In considering unmeasured factors, it is notable that close matches were found from patients referred and considered for ECMO, but subsequently treated conventionally. Unmeasured features that may have influenced contemporaneous decision-making include views expressed over a telephone consult that are not reflected in available data. We note that characteristics and outcomes of referred patients changed over the course of our study period, and it is likely that understanding of patients thought to benefit from ECMO evolved over time. To challenge this, we restricted patient population in sensitivity analysis using human assigned labels ("met NHS criteria"), finding a reduced, but still considerable, ARR of $14.5 \%$. The use of case-based matching also served to minimise temporal bias, with each case closely matched across clinical and physiological parameters. $E$ values across our analyses suggest that substantial unmeasured confounding would be required to negate the observed treatment effect [23].

It is also important to acknowledge that realities of unprecedented pandemic demands may account for some of these close matches. It is reasonable to assume that some patients who could have benefited from ECMO may not have received it. The UK doubled its maximum ECMO capacity, indicative of significant system load, and it is conceivable that decision-making for cases with marginal or unclear benefit could 
Table 2 Characteristics and outcomes of conventionally and ECMO-treated patients before and after GenMatch matching, showing all co-variates used in matching procedure

\begin{tabular}{|c|c|c|c|c|c|c|c|c|}
\hline & \multicolumn{4}{|l|}{ Before matching } & \multicolumn{4}{|c|}{ After GenMatch matching } \\
\hline & $\begin{array}{l}\text { Conventional } \\
(n=690)\end{array}$ & $\begin{array}{l}\text { ECMO } \\
(n=243)\end{array}$ & SMD & $p$-value & $\begin{array}{l}\text { Conventional } \\
(n=209)\end{array}$ & $\begin{array}{l}\text { ECMO } \\
(n=209)\end{array}$ & SMD & $p$-value \\
\hline Propensity score & $0.14[0.06,0.3]$ & $0.41[0.27,0.59]$ & 1.13 & $<0.001$ & $0.37[0.24,0.49]$ & $0.38[0.24,0.5]$ & 0.03 & 0.733 \\
\hline \multicolumn{9}{|l|}{ Demographics } \\
\hline Age (years) & $53[45,59]$ & $46[39,52]$ & -0.7 & $<0.001$ & $48[39,55]$ & $46[40,53]$ & -0.05 & 0.416 \\
\hline Male sex & $474(68.7)$ & $167(68.7)$ & 0 & 1 & $146(69.9)$ & $144(68.9)$ & -0.02 & 0.915 \\
\hline $\mathrm{BMI}\left(\mathrm{kg} / \mathrm{m}^{2}\right)$ & $31.24[27.04,35.64]$ & $31.67[28.17,35.76]$ & -0.01 & 0.433 & $31.84[27.76,35.8]$ & $31.64[28.37,35.92]$ & -0.04 & 0.868 \\
\hline Clinical frailty scale & $2[2,2]$ & $2[1,2]$ & -0.34 & $<0.001$ & $2[1,2]$ & $2[1,2]$ & 0.02 & 0.824 \\
\hline $\begin{array}{l}\text { Immuno-compro- } \\
\text { mised }\end{array}$ & $35(5.1)$ & $5(2.1)$ & -0.21 & 0.07 & $4(1.9)$ & $4(1.9)$ & 0 & 1 \\
\hline \multicolumn{9}{|c|}{ Pre-referral admission characteristics } \\
\hline $\begin{array}{l}\text { Hospital duration } \\
\text { prior to IMV (days) }\end{array}$ & $4.53[1.7,7.99]$ & $4.16[2,7.2]$ & 0.03 & 0.81 & $4.66[2,7.6]$ & $4.11[2,7.27]$ & 0.02 & 0.94 \\
\hline Duration of IMV (days) & $3.16[1,6]$ & $2[1,5]$ & -0.48 & $<0.001$ & $2[1,4]$ & $2[1,5]$ & 0.1 & 0.239 \\
\hline Cardiac arrest & $19(2.8)$ & $5(2.1)$ & -0.05 & 0.724 & $4(1.9)$ & $5(2.4)$ & 0.03 & 1 \\
\hline \multicolumn{9}{|c|}{ Therapeutic interventions } \\
\hline NIV & $444(64.3)$ & $168(69.1)$ & 0.1 & 0.203 & $152(72.7)$ & $146(69.9)$ & -0.06 & 0.589 \\
\hline Corticosteroids & $463(67.1)$ & $143(58.8)$ & -0.17 & 0.025 & $134(64.1)$ & $129(61.7)$ & -0.05 & 0.685 \\
\hline IL-6 inhibitor & $61(8.8)$ & $16(6.6)$ & -0.09 & 0.335 & $11(5.3)$ & $15(7.2)$ & 0.08 & 0.543 \\
\hline Prone position trial & $519(75.2)$ & $193(79.4)$ & 0.1 & 0.215 & $171(81.8)$ & $163(78)$ & -0.09 & 0.393 \\
\hline $\begin{array}{l}\text { Chest drain(s) for } \\
\text { pneumothorax }\end{array}$ & $41(5.9)$ & $14(5.8)$ & -0.01 & 1 & $9(4.3)$ & $11(5.3)$ & 0.04 & 0.819 \\
\hline RRT & $57(8.3)$ & $6(2.5)$ & -0.37 & 0.003 & $4(1.9)$ & $6(2.9)$ & 0.06 & 0.749 \\
\hline Anticoagulation & & & & 0.093 & & & & 1 \\
\hline Contraindicated & $7(1)$ & $0(0)$ & -0.12 & & $0(0)$ & $0(0)$ & 0 & \\
\hline Prophylactic & $468(67.8)$ & $179(73.7)$ & 0.13 & & $151(72.2)$ & $151(72.2)$ & 0 & \\
\hline Therapeutic & $215(31.2)$ & $64(26.3)$ & -0.11 & & $58(27.8)$ & $58(27.8)$ & 0 & \\
\hline \multicolumn{9}{|c|}{ Respiratory parameters at time of referral } \\
\hline P/F ratio $(\mathrm{mmHg})$ & $84[69.98,105.62]$ & $71.55[61.5,83.7]$ & -0.83 & $<0.001$ & $76.50[63.44,86.72]$ & $72.53[63,85.61]$ & -0.03 & 0.385 \\
\hline $\begin{array}{l}\text { Static compliance } \\
\left(\mathrm{mL} / \mathrm{cmH}_{2} \mathrm{O}\right)\end{array}$ & $30[22.11,37.5]$ & $29.36[21.62,34.71]$ & -0.04 & 0.339 & $30[21.86,35.25]$ & $29.23[21.58,35]$ & 0.09 & 0.654 \\
\hline $\begin{array}{l}\text { Driving pressure } \\
\left(\mathrm{cmH}_{2} \mathrm{O}\right)\end{array}$ & $15.56[13,19]$ & $16[13,20]$ & 0.03 & 0.483 & $16[14,19.4]$ & $16[13,20]$ & -0.02 & 0.619 \\
\hline $\begin{array}{l}\text { Plateau pressure } \\
\left(\mathrm{cmH}_{2} \mathrm{O}\right)\end{array}$ & $29[25,31]$ & $29[25,31.69]$ & 0.03 & 0.617 & $29[26,31]$ & $29[25,32]$ & -0.05 & 0.843 \\
\hline Vt/PBW (mL/kg) & $6.72[6.01,7.99]$ & $6.79[6.14,7.88]$ & 0.03 & 0.613 & $6.69[6.14,7.99]$ & $6.73[6.1,7.85]$ & 0 & 0.883 \\
\hline PEEP $\left(\mathrm{cmH}_{2} \mathrm{O}\right)$ & $12[10,14.43]$ & $12[10,14]$ & -0.08 & 0.416 & $12[10,14.18]$ & $12[10,14]$ & -0.04 & 0.716 \\
\hline $\begin{array}{l}\text { Minute ventilation } \\
(\mathrm{L} / \mathrm{min})\end{array}$ & $9.85[8,11.51]$ & $10[8.13,11.41]$ & 0.06 & 0.707 & $9.91[8.17,11.58]$ & $9.88[8.1,11.39]$ & -0.01 & 0.62 \\
\hline \multicolumn{9}{|c|}{ Arterial blood gas parameters at time of referral } \\
\hline $\mathrm{pH}$ & $7.32[7.25,7.39]$ & $7.31[7.25,7.38]$ & -0.1 & 0.288 & $7.31[7.25,7.39]$ & $7.31[7.24,7.38]$ & -0.08 & 0.962 \\
\hline $\mathrm{pCO}_{2}(\mathrm{mmHg})$ & $58.2[48.94,68.33]$ & $57.9[49.88,69]$ & 0.06 & 0.527 & $58.5[50.25,68.33]$ & $58.5[50.4,68.25]$ & 0.07 & 0.732 \\
\hline Base excess (mmol/L) & $3.7[1.8,7.77]$ & $3.3[1.65,6.34]$ & -0.18 & 0.157 & $3[1.7,6.3]$ & $3.57[1.7,6.4]$ & 0.01 & 0.349 \\
\hline Lactate $(\mathrm{mmol} / \mathrm{L})$ & $1.3[1,1.7]$ & $1.31[1.1,1.8]$ & 0.04 & 0.209 & $1.3[1,1.6]$ & $1.30[1.1,1.77]$ & 0.08 & 0.177 \\
\hline \multicolumn{9}{|c|}{ Laboratory parameters at time of referral } \\
\hline $\begin{array}{l}\text { White cell count } \\
\left(10^{9} / \mathrm{L}\right)\end{array}$ & $11.68[9,15.08]$ & $12[9.71,14.8]$ & 0.02 & 0.498 & $11.70[9.3,14.8]$ & $12[9.7,14.7]$ & 0.08 & 0.68 \\
\hline Platelet count $\left(10^{9} / \mathrm{L}\right)$ & $\begin{array}{l}273.94[228 \\
321.75]\end{array}$ & $\begin{array}{l}283.91[254.77, \\
332.16]\end{array}$ & 0.26 & 0.001 & $290[249.34,343.87]$ & $283.91[253,331.59]$ & -0.05 & 0.626 \\
\hline Haemoglobin (g/L) & $115[101,126.96]$ & $116[101,127]$ & 0.07 & 0.539 & $119[107,129]$ & $117[101,127]$ & -0.06 & 0.256 \\
\hline
\end{tabular}


Table 2 (continued)

\begin{tabular}{|c|c|c|c|c|c|c|c|c|}
\hline & \multicolumn{4}{|l|}{ Before matching } & \multicolumn{4}{|c|}{ After GenMatch matching } \\
\hline & $\begin{array}{l}\text { Conventional } \\
(n=690)\end{array}$ & $\begin{array}{l}\text { ECMO } \\
(n=243)\end{array}$ & SMD & $p$-value & $\begin{array}{l}\text { Conventional } \\
(n=209)\end{array}$ & $\begin{array}{l}\text { ECMO } \\
(n=209)\end{array}$ & SMD & $p$-value \\
\hline $\begin{array}{l}\text { C-reactive protein } \\
(\mathrm{mg} / \mathrm{L})\end{array}$ & $159.4[71,262.85]$ & $192[104,299.5]$ & 0.23 & 0.001 & $170[81,280]$ & $187[101,296.61]$ & 0.08 & 0.314 \\
\hline \multicolumn{9}{|c|}{ Severity and risk scores } \\
\hline RESP score & $4[3,5]$ & $5[4,6]$ & 0.81 & $<0.001$ & $5[4,6]$ & $5[4,6]$ & -0.09 & 0.175 \\
\hline SOFA score & $5[4,7]$ & $5[4,7.02]$ & -0.02 & 0.363 & $4.96[4,7]$ & $5[4,7.04]$ & 0.08 & 0.427 \\
\hline \multicolumn{9}{|l|}{ Outcomes } \\
\hline Died & 343 (49.7) & $60(24.7)$ & & $<0.001$ & $92(44)$ & $54(25.8)$ & & $<0.001$ \\
\hline
\end{tabular}

Values are expressed as median value $[95 \% \mathrm{Cl}]$ or as $\mathrm{n}(\%) ; B M I$ body mass index, IMV invasive mechanical ventilation, $N I V$ non-invasive ventilation, $R R T$ renal replacement therapy, $P / F$ ratio partial pressure of arterial oxygen to fraction of inspired oxygen ratio, $V t$ tidal volume, $P E E P$ positive end expiratory pressure, $P B W$ predicted body weight, RESP respiratory ECMO survival prediction, SOFA sequential organ failure assessment

have been influenced by factors related to resource availability at referring or receiving centre.

This study builds on previous studies of ECMO in H1N1 pandemic influenza [33, 34]. Our pool of eligible non-ECMO patients for matching was larger: $n=690$ vs $n=195$ (Noah et al.) and $n=157$ (Pham et al.), resulting in more matches $(n=209$ out of 243 eligible). Additionally, referrals recorded detailed pre-ECMO data; thus, matching was performed using variables that might represent heterogenous phenotypes [35, 36]. In designing this study, we address potential limitations discussed by Pham et al.: incorporation of rich covariates, reporting of matching procedures, use of stringent calliper width, and matching without replacement.

Our study presents several strengths, including use of rich covariate data to control most patientcentric factors. Our results remained robust through sensitivity analyses. ECMO patients were selected through the same nationally defined pathway and, outside of the concerns discussed, present a welldefined treatment group (previous study populations were treated in numerous centres with heterogeneous ECMO criteria). The use of data on which real-world decision-making took place adds pragmatism, and interpretability.

We also note important limitations. First, the possibility of residual confounding, which has been discussed. Second, pre-selection of patients via national referral criteria, and mixed exposure of both ECMO and SRF centre treatment, may limit generalisability to international cohorts where pathways differ. Third, we did not introduce measures of hospital-level strain or differences in conventional management, potentially resulting in exposure heterogeneity (i.e. transfer of patients from a 'high strain' hospital to a lower strain' hospital or varying application of conventional management). However, analysis of UK hospital-level variation provides little evidence of differing strain between sites $[37,38]$ in context of nation-wide measures to 'offload' hospitals via patient transfer services [39]. Fourth, analysis is limited to in-hospital mortality, without examination of delayed out-of-hospital mortality, functional status of survivors or secondary complications on ECMO that may contribute to patient morbidity. Fifth, despite consistent results in sensitivity analysis for case-based data missingness, imputation of high missing for some variables (with assumption of missing-at-random) may reduce robustness. In summary, while similar outcomes were achieved across multiple analyses, results should be interpreted with caution.

\section{Conclusion}

Our study suggests that ECMO provided at specialist centres offers a survival benefit in selected patients with COVID-19 SRF, compared to similar patients treated with conventional therapy in their local hospital. Where resources and specialisation allow, ECMO should be offered in this critically unwell patient group. The subset of patients with severe COVID-19 who are likely to benefit most from ECMO remains unclear, and further work is required to determine factors which can assist in the decisionmaking process. 
Table 3 Comparison of matched (by GenMatch) and unmatched ECMO-treated patients

\begin{tabular}{|c|c|c|c|}
\hline & Unmatched $(n=34)$ & Matched $(n=209)$ & $p$-value \\
\hline \multicolumn{4}{|l|}{ Demographics } \\
\hline Age (years) & $37[34,45.75]$ & $46[40,53]$ & $<0.001$ \\
\hline Male sex & $23(67.6)$ & $144(68.9)$ & 1 \\
\hline Body mass index $\left(\mathrm{kg} / \mathrm{m}^{2}\right)$ & $31.77[26.84,34.05]$ & $31.64[28.37,35.92]$ & 0.559 \\
\hline Clinical frailty scale & $2[1,2]$ & $2[1,2]$ & 0.013 \\
\hline Immuno-compromised & $1(2.9)$ & $4(1.9)$ & 1 \\
\hline \multicolumn{4}{|l|}{ Pre-referral admission characteristics } \\
\hline Hospital duration prior to IMV (days) & $5[2.76,6.49]$ & $4.11[2,7.27]$ & 0.52 \\
\hline Duration of IMV (days) & $1.5[1,3]$ & $2[1,5]$ & 0.021 \\
\hline Cardiac arrest & $0(0)$ & $5(2.4)$ & 0.795 \\
\hline \multicolumn{4}{|l|}{ Therapeutic interventions } \\
\hline Non-invasive ventilation & $22(64.7)$ & $146(69.9)$ & 0.687 \\
\hline Corticosteroids & $14(41.2)$ & $129(61.7)$ & 0.038 \\
\hline Interleukin-6 inhibitor & $1(2.9)$ & $15(7.2)$ & 0.582 \\
\hline Prone position trial & $30(88.2)$ & $163(78)$ & 0.254 \\
\hline Chest drain(s) for pneumothorax & $3(8.8)$ & $11(5.3)$ & 0.668 \\
\hline Renal replacement therapy & $0(0)$ & $6(2.9)$ & 0.686 \\
\hline Anticoagulation & & & 0.303 \\
\hline Contraindicated & $0(0)$ & $0(0)$ & \\
\hline Prophylactic & $28(82.4)$ & $151(72.2)$ & \\
\hline Therapeutic & $6(17.6)$ & $58(27.8)$ & \\
\hline \multicolumn{4}{|l|}{ Respiratory parameters at time of referral } \\
\hline P/F ratio $(\mathrm{mmHg})$ & $62.62[57.36,71.63]$ & $72.53[63,85.61]$ & $<0.001$ \\
\hline Static compliance $\left(\mathrm{mL} / \mathrm{cmH}_{2} \mathrm{O}\right)$ & $31.73[25.18,33.21]$ & $29.23[21.58,35]$ & 0.404 \\
\hline Driving pressure $\left(\mathrm{cmH}_{2} \mathrm{O}\right)$ & $16[14.18,20]$ & $16[13,20]$ & 0.765 \\
\hline Plateau pressure $\left(\mathrm{cmH}_{2} \mathrm{O}\right)$ & $28.5[25.25,30]$ & $29[25,32]$ & 0.414 \\
\hline $\mathrm{Vt} / \mathrm{PBW}(\mathrm{mL} / \mathrm{kg})$ & $6.92[6.56,7.99]$ & $6.73[6.1,7.85]$ & 0.266 \\
\hline Positive end expiratory pressure $\left(\mathrm{cmH}_{2} \mathrm{O}\right)$ & $11.96[10,14]$ & $12[10,14]$ & 0.213 \\
\hline Minute ventilation (L/min) & $10.38[9,11.71]$ & $9.88[8.1,11.39]$ & 0.255 \\
\hline \multicolumn{4}{|c|}{ Arterial blood gas parameters at time of referral } \\
\hline $\mathrm{pH}$ & $7.31[7.25,7.36]$ & $7.31[7.24,7.38]$ & 0.69 \\
\hline $\mathrm{pCO}_{2}(\mathrm{mmHg})$ & $54.25[47.46,71.46]$ & $58.5[50.4,68.25]$ & 0.475 \\
\hline Base excess (mmol/L) & $2.77[1.39,5.18]$ & $3.57[1.7,6.4]$ & 0.166 \\
\hline Lactate $(\mathrm{mmol} / \mathrm{L})$ & $1.32[1.07,2.1]$ & $1.3[1.1,1.77]$ & 0.406 \\
\hline \multicolumn{4}{|l|}{ Laboratory parameters at time of referral } \\
\hline White cell count $\left(10^{9} / \mathrm{L}\right)$ & $12[9.74,15.23]$ & $12[9.7,14.7]$ & 0.829 \\
\hline Platelet count $\left(10^{9} / \mathrm{L}\right)$ & $285.78[266.5,363.49]$ & $283.91[253,331.59]$ & 0.515 \\
\hline Haemoglobin $(\mathrm{g} / \mathrm{L})$ & $109.5[100.25,125.75]$ & $117[101,127]$ & 0.406 \\
\hline C-reactive protein (mg/L) & $240.9[152,332.75]$ & $187[101,296.61]$ & 0.133 \\
\hline \multicolumn{4}{|l|}{ Severity and risk scores } \\
\hline RESP score & $5[5,7]$ & $5[4,6]$ & 0.011 \\
\hline SOFA score & $6.61[4.08,7]$ & $5[4,7.04]$ & 0.205 \\
\hline \multicolumn{4}{|l|}{ Outcomes } \\
\hline Died & $6(17.6)$ & $54(25.8)$ & 0.416 \\
\hline
\end{tabular}

Values are expressed as median value $[95 \% \mathrm{Cl}]$ or as $\mathrm{n}(\%)$; IMV invasive mechanical ventilation, $P / F$ ratio partial pressure of arterial oxygen to fraction of inspired oxygen ratio, $V t$ tidal volume, $P B W$ predicted body weight, RESP respiratory ECMO survival prediction, SOFA sequential organ failure assessment 


\section{Supplementary Information}

The online version contains supplementary material available at https://doi. org/10.1007/s00134-022-06645-w.

\section{Author details}

${ }^{1}$ Department of Adult Critical Care, Guy's and St Thomas' NHS Foundation Trust, London, UK. ${ }^{2}$ Department of Critical Care, King's College Hospital NHS Foundation Trust, London, UK. ${ }^{3}$ Institute of Global Health Innovation, Imperial College London, London, UK. ${ }^{4}$ Department of Adult Intensive Care, Royal Brompton and Harefield NHS Foundation Trust, London, UK. ${ }^{5}$ Division of Asthma, Allergy and Lung Biology, King's College London, London, UK. ${ }^{6}$ Department of Adult Critical Care, St Thomas' Hospital, London SE1 7EH, UK.

\section{Acknowledgements}

We thank Vagish Kumar and the ECMO specialist nurses at St. Thomas' Hospital for their contribution to data collection. We thank all staff in referring hospitals and ECMO centers who have gone above and beyond in caring for COVID-19 patients during the extremely trying times created by the COVID-19 pandemic. We thank all those involved in accurately documenting these referrals and responding to follow up requests, without whom this analysis would not have been possible.

\section{Author contributions}

SW and JZ were responsible for study conception and methodology. SW, JZ, $\mathrm{RL}$ and $\mathrm{MB}$ were responsible for data collection and cleaning. SW and JZ were responsible for analysis. All authors were responsible for manuscript writing and editing.

\section{Declarations}

\section{Conflicts of interest}

This study received no direct funding. JZ receives funding from the Wellcome Trust (203928/Z/16/Z) and acknowledges support from the National Institute for Health Research (NIHR) Biomedical Research Centre based at Imperial College NHS Trust and Imperial College London. Other authors have no financial or non-financial competing interests to declare.

\section{Ethical approval}

This study qualified as a service evaluation, as defined by the UK Health Research Authority, with waiver of individual informed consent. Data collection and analysis were carried out under evaluation for national ECMO commissioning.

\section{Publisher's Note}

Springer Nature remains neutral with regard to jurisdictional claims in published maps and institutional affiliations.

\section{Received: 29 November 2021 Accepted: 7 February 2022} Published: 3 March 2022

\section{References}

1. Falcoz P-E, Monnier A, Puyraveau M et al (2020) Extracorporeal membrane oxygenation for critically ill patients with COVID-19 related acute respiratory distress syndrome: worth the effort? Am J Respir Crit Care Med. https://doi.org/10.1164/rccm.202004-1370LE

2. Henry BM, Lippi G (2020) Poor survival with extracorporeal membrane oxygenation in acute respiratory distress syndrome (ARDS) due to coronavirus disease 2019 (COVID-19): pooled analysis of early reports. J Crit Care 58:27-28. https://doi.org/10.1016/j.jcrc.2020.03.011

3. Lebreton G, Schmidt M, Ponnaiah M et al (2021) Extracorporeal membrane oxygenation network organisation and clinical outcomes during the COVID-19 pandemic in Greater Paris, France: a multicentre cohort study. Lancet Respir Med 9:851-862. https://doi.org/10.1016/S22132600(21)00096-5

4. Karagiannidis C, Slutsky AS, Bein T et al (2021) Complete countrywide mortality in COVID patients receiving ECMO in Germany throughout the first three waves of the pandemic. Crit Care 25:413. https://doi.org/10. 1186/s13054-021-03831-y

5. Schmidt M, Hajage D, Lebreton G et al (2020) Extracorporeal membrane oxygenation for severe acute respiratory distress syndrome associated with COVID-19: a retrospective cohort study. Lancet Respir Med. https:// doi.org/10.1016/S2213-2600(20)30328-3

6. Zhang J, Merrick B, Correa GL et al (2020) Veno-venous extracorporeal membrane oxygenation in coronavirus disease 2019: a case series. ERJ Open Research. https://doi.org/10.1183/23120541.00463-2020

7. Ramanathan K, Shekar K, Ling RR et al (2021) Extracorporeal membrane oxygenation for COVID-19: a systematic review and meta-analysis. Crit Care 25:211. https://doi.org/10.1186/s13054-021-03634-1

8. Bellani G, Laffey JG, Pham T et al (2016) Epidemiology, patterns of care, and mortality for patients with acute respiratory distress syndrome in intensive care units in 50 countries. JAMA 315:788. https://doi.org/10. 1001/jama.2016.0291

9. Shekar K, Badulak J, Peek G et al (2020) Extracorporeal life support organization coronavirus disease 2019 interim guidelines: a consensus document from an international group of interdisciplinary extracorporeal membrane oxygenation providers. ASAIO J 66:707-721. https://doi.org/ 10.1097/MAT.00000000000001193

10. Badulak J, Antonini MV, Stead CM et al (2021) Extracorporeal membrane oxygenation for COVID-19: updated 2021 guidelines from the extracorporeal life support organization. ASAIO J 67:485-495. https://doi.org/10. 1097/MAT.0000000000001422

11. Fang J, Li R, Chen Y et al (2021) Extracorporeal membrane oxygenation therapy for critically ill coronavirus disease 2019 patients in Wuhan, China: a retrospective multicenter cohort study. Curr Med Sci 41:1-13. https:// doi.org/10.1007/s11596-021-2311-8

12. The STOP-COVID Investigators, Shaefi S, Brenner SK et al (2021) Extracorporeal membrane oxygenation in patients with severe respiratory failure from COVID-19. Intensive Care Med 47:208-221. https://doi.org/10.1007/ s00134-020-06331-9

13. Haiduc AA, Alom S, Melamed N, Harky A (2020) Role of extracorporeal membrane oxygenation in COVID-19: a systematic review. J Card Surg 35:2679-2687. https://doi.org/10.1111/jocs.14879

14. NHS England (2020) Clinical guide for extra corporeal membrane oxygenation (ECMO) for respiratory failure in adults during the coronavirus pandemic. https://warwick.ac.uk/fac/sci/med/research/ctu/trials/recov ery-rs/news/speciality-guide-extra-corporealmembrane-oxygenationecmo-adult.pdf

15. Camporota L, Meadows C, Ledot S et al (2021) Consensus on the referral and admission of patients with severe respiratory failure to the NHS ECMO service. Lancet Respir Med 9:e16-e17. https://doi.org/10.1016/ S2213-2600(20)30581-6

16. Czapran A, Steel M, Barrett NA (2020) Extra-corporeal membrane oxygenation for severe respiratory failure in the UK. J Intensive Care Soc 21:247-255. https://doi.org/10.1177/1751143719870082

17. NHS England (2019) Adult ECMO service specification. https://www.engla nd.nhs.uk/wp-content/uploads/2019/02/Adult-ECMO-Service-Specificat ion.pdf

18. Peek GJ, Mugford M, Tiruvoipati R et al (2009) Efficacy and economic assessment of conventional ventilatory support versus extracorporeal membrane oxygenation for severe adult respiratory failure (CESAR): a multicentre randomised controlled trial. Lancet 374:1351. https://doi.org/ 10.1016/S0140-6736(09)61069-2

19. Stekhoven DJ, Buhlmann P (2012) MissForest—non-parametric missing value imputation for mixed-type data. Bioinformatics 28:112-118. https:// doi.org/10.1093/bioinformatics/btr597

20. Franchetti $Y$ (2021) Use of propensity scoring and its application to real world data: advantages, disadvantages, and methodological objectives explained to researchers without using mathematical equations. J Clin Pharmacol. https://doi.org/10.1002/jcph.1989

21. Diamond A, Sekhon JS (2013) Genetic matching for estimating causal effects: a general multivariate matching method for achieving balance in observational studies. Rev Econ Stat 95:932-945. https://doi.org/10.1162/ REST_a_00318

22. Written on Behalf of AME Big-Data Clinical Trial Collaborative Group, Zhang Z, Kim HJ et al (2019) Balance diagnostics after propensity score matching. Ann Transl Med 7:16-16. https://doi.org/10.21037/atm.2018. 12.10 
23. VanderWeele TJ, Ding P (2017) Sensitivity analysis in observational research: introducing the E-value. Ann Intern Med 167:268-274. https:// doi.org/10.7326/M16-2607

24. Mathur MB, Ding P, Riddell CA, VanderWeele TJ (2018) Web site and R package for computing E-values. Epidemiology 29:e45-e47. https://doi. org/10.1097/EDE.0000000000000864

25. Ho DE, Imai K, King G, Stuart EA (2011) Matchlt: nonparametric preprocessing for parametric causal inference. J Stat Soft. https://doi.org/10. 18637/jss.v042.i08

26. Barbaro RP, MacLaren G, Boonstra PS et al (2020) Extracorporeal membrane oxygenation support in COVID-19: an international cohort study of the Extracorporeal Life Support Organization registry. Lancet 396:10711078. https://doi.org/10.1016/S0140-6736(20)32008-0

27. Combes A, Hajage D, Capellier G et al (2018) Extracorporeal membrane oxygenation for severe acute respiratory distress syndrome. N Engl J Med 378:1965-1975. https://doi.org/10.1056/NEJMoa1800385

28. Gattinoni L, Vasques F, Quintel M (2018) Use of ECMO in ARDS: does the EOLIA trial really help? Crit Care 22:171. https://doi.org/10.1186/ s13054-018-2098-6

29. Sameed M, Meng Z, Marciniak ET (2019) EOLIA trial: the future of extracorporeal membrane oxygenation in acute respiratory distress syndrome therapy? Breathe 15:244-246. https://doi.org/10.1183/20734 735.0363-2018

30. Barbaro RP, MacLaren G, Boonstra PS et al (2021) Extracorporeal membrane oxygenation for COVID-19: evolving outcomes from the international Extracorporeal Life Support Organization Registry. The Lancet 398:1230-1238. https://doi.org/10.1016/S0140-6736(21)01960-7

31. Riera J, Roncon-Albuquerque R, Fuset MP et al (2021) Increased mortality in patients with COVID-19 receiving extracorporeal respiratory support during the second wave of the pandemic. Intensive Care Med. https:// doi.org/10.1007/s00134-021-06517-9
32. Riera J, Alcántara S, Bonilla C et al (2021) Risk factors for mortality in patients with COVID-19 needing extracorporeal respiratory support. Eur Respir J. https://doi.org/10.1183/13993003.02463-2021

33. Pham T, Combes A, Rozé H et al (2013) Extracorporeal membrane oxygenation for pandemic influenza A ( $\mathrm{H} 1 \mathrm{~N} 1)$-induced acute respiratory distress syndrome: a cohort study and propensity-matched analysis. Am J Respir Crit Care Med 187:276-285. https://doi.org/10.1164/rccm. 201205-08150C

34. Noah MA, Peek GJ, Finney SJ et al (2011) Referral to an extracorporeal membrane oxygenation center and mortality among patients with severe 2009 influenza A (H1N1). JAMA 306:1659. https://doi.org/10.1001/ jama.2011.1471

35. Zhang J, Whebell SF, Sanderson B et al (2021) Phenotypes of severe COVID-19 ARDS receiving extracorporeal membrane oxygenation. $\mathrm{Br} J$ Anaesth 126:e130-e132. https://doi.org/10.1016/j.bja.2020.12.023

36. Azoulay E, Zafrani L, Mirouse A et al (2020) Clinical phenotypes of critically ill COVID-19 patients. Intensive Care Med 46:1651-1652. https://doi. org/10.1007/s00134-020-06120-4

37. Bottle A, Faitna P, Aylin PP (2021) Patient-level and hospital-level variation and related time trends in COVID-19 case fatality rates during the first pandemic wave in England: multilevel modelling analysis of routine data. BMJ Qual Saf. https://doi.org/10.1136/bmjqs-2021-012990

38. Gray WK, Navaratnam AV, Day J et al (2021) Variability in COVID-19 in-hospital mortality rates between national health service trusts and regions in England: a national observational study for the Getting It Right First Time Programme. EClinicalMedicine 35:100859. https://doi.org/10. 1016/j.eclinm.2021.100859

39. NHS England (2021) Service specification for adult critical care transfer services. https://www.england.nhs.uk/wp-content/uploads/2021/06/ Service-Specification-Adult-Critical-Care-Transfer-services.pdf 